\title{
Rectifying fluctuations in an optical lattice
}

\author{
P.H. Jones, M. Goonasekera and F. Renzoni \\ Departement of Physics and Astronomy, University College London, \\ Gower Street, London WC1E 6BT, United Kingdom
}

(Dated: June 30, 2018)

\begin{abstract}
We have realized a Brownian motor by using cold atoms in a dissipative optical lattice as a model system. In our experiment the optical potential is spatially symmetric and the time-symmetry of the system is broken by applying appropriate zero-mean ac forces. We identify a regime of rectification of forces and a regime of rectification of fluctuations, the latter corresponding to the realization of a Brownian motor.

PACS numbers: 05.45.-a, 42.65.Es, 32.80.Pj
\end{abstract}

Noise is unavoidably present in every physical, chemical and biological process. From electronic devices to laser action, from chemical reactions to the motion of bacteria, noise modifies the process by introducing random fluctuations in the observed dynamics.

Noise is often treated as a nuisance to be reduced as much as possible. Consider for example an electronic device, typically an amplifier, with an input and an output. It is often believed that the only way to improve the signal-to-noise ratio (SNR) at the output of the device is to reduce the noise at its input. This is not necessarily true: for a nonlinear device the provision of additional noise to the input signal may increase the SNR of the output signal, a phenomenon known as stochastic resonance and observed in a variety of processes in electronics, physics, chemistry and biology [1].

Noise also plays a central role in Brownian motors [2], the topic of the present work, which recently attracted much interest $3,[4,[5,6,7,68,9]$ as it is believed that they may constitute a model for biological molecular motors [10]. Consider a sample of Brownian particles diffusing through a periodic potential in the presence of oscillating forces of zero average. A net current of particles can be induced by breaking the symmetry of the system, realizing in this way the somewhat surprising situation of directed motion in a macroscopically flat potential in the absence of applied bias forces. Whenever the net current of particles arises from the rectification of fluctuations the directed motion corresponds to the realisation of a so-called Brownian motor.

In this work we realize a Brownian motor by using cold atoms in a dissipative optical lattice as a model system. The optical potential is spatially symmetric, and the time-symmetry of the system is broken by applying appropriate zero-mean ac forces. We identify a regime of rectification of the fluctuations, corresponding to the realization of a Brownian motor, and a regime of rectification of the forces. In the regime of rectification of the fluctuations, the current amplitude vanishes in the absence of noise, and shows a stochastic resonance-like behavior at increasing noise amplitude, therefore confirming that our device acts as a fluctuations rectifier. In the regime of rectification of the forces, the directed motion of the atoms through the lattice is only due to deterministic forces. In this regime the noise acts as a nuisance and correspondingly the current decreases for increasing amplitude of the noise.

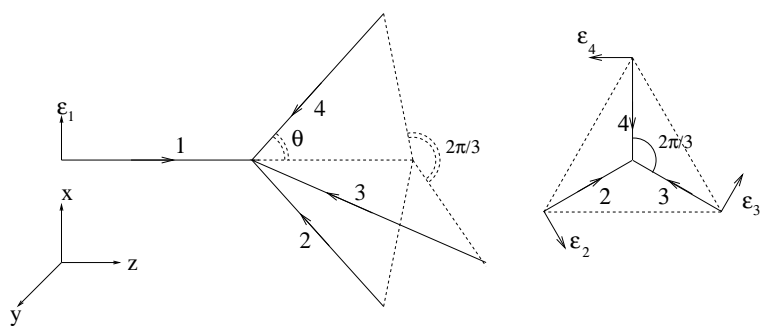

FIG. 1: Lattice beams configuration. Left: arrangement of the four beams in the umbrellalike configuration. Only the polarization of beam 1 is shown. Right: polarizations of beams $2-4$ as seen by looking towards the $-z$ direction.

The periodic potential used in this work is a $3 \mathrm{D}$ optical lattice [11] determined by the interference of four laser beams (beams $1-4$, with wavevectors $\vec{k}_{1}-\vec{k}_{4}$ ), arranged in the so-called umbrellalike configuration [12], as sketched in Fig. 11 One laser beam (beam 1) propagates in the $z$-direction. The three other laser beams propagate in the opposite direction, and are arranged along the edges of a triangular pyramid having the $z$-direction as axis, with the azimuthal angle between each pair of beams equal to $2 \pi / 3$. All beams are linearly polarized, with the polarization of beam 1 in the $x$-direction. The linear polarization of beam $j$ is chosen as $\vec{\epsilon}_{j}=\vec{k}_{1} \wedge \vec{k}_{j} / k^{2}$, i.e. $\vec{\epsilon}_{j}$ is orthogonal to the plane defined by $\vec{k}_{1}$ and $\vec{k}_{j}$ and ori-

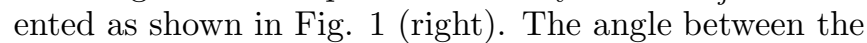
beam $j$, with $j=2-4$, and the $z$ axis is $\theta=30^{\circ}$, and we have made the following choice for the fields amplitudes: $E_{1}=\mathcal{E}_{0}, E_{2}=E_{3}=\mathcal{E}_{0} \sqrt{3+\cos ^{2} \theta} / 6 \cos \theta=\mathcal{E}_{0} \sqrt{5} / 6$ and $E_{4}=\mathcal{E}_{0} / 3$. The interference of the laser fields produces a periodic and spatially symmetric optical potential, with the potential minima associated with pure circular $\left(\sigma^{+}\right.$or $\left.\sigma^{-}\right)$polarization of the light 12]. For an atom with a $F_{g}=F \rightarrow F_{e}=F+1$ transition, the optical potential consists precisely of $2 F+1$ potentials, one for each ground state sublevel of the atom. Transitions between different potentials are produced by optical pumping processes, which transfer an atom from one 
ground state sublevel to another one. As optical pumping is a stochastic process, this introduces fluctuations in the atomic dynamics. These fluctuations result in a random walk through the optical lattice, and indeed normal diffusion has been observed for the atomic cloud expanding in the lattice for a broad range of interaction parameters 11].

In a spatially symmetric structure directed motion can be induced by breaking the time-symmetry of the system [4]. With this aim in mind, we apply a zero-mean ac force composed of two harmonics

$$
F(t)=F_{0}[A \cos (\omega t)+B \cos (2 \omega t-\phi)] .
$$

For $\phi \neq n \pi$, with $n$ integer, the force $F(t)$ breaks the time-symmetry of the system. To be precise, for an arbitrary choice of the phase $\phi$ the force $F(t)$ breaks the generalized-parity symmetry $(x, p, t) \rightarrow(-x,-p, t+$ $\pi / \omega)$, and for $\phi \neq n \pi$ the residual time-reversal symme$\operatorname{try}(x, t) \rightarrow(x,-p,-t)$ is also broken [4]. Experimentally, to introduce a homogenous time-dependent force we apply a phase modulation to the beam 1 of the form

$$
\alpha(t)=\alpha_{0}\left[A \cos (\omega t)+\frac{B}{4} \cos (2 \omega t-\phi)\right] .
$$

In the accelerated frame in which the optical lattice is stationary the phase modulation results in an inertial force $F$ of the form of Eq. 11 with $F_{0}=m \omega^{2} \alpha_{0} / 2 k$, where $m$ is the atomic mass [13]. In the present work, the phase difference between the two harmonics is kept fixed at $\phi=\pi / 2$, so as to break the time-symmetry of the system and induce directed motion. The coefficients $A$ and $B$ of the two harmonics will be taken as equal: $A=B=1$.

Our system differs from the usual models for Brownian motors mainly in one respect: here we do not have a single potential, but a potential for each ground state of the atom, and both fluctuations in the atomic dynamics and the friction are associated with optical pumping between ground states, i.e. optical pumping between different optical potentials 11]. Because of this difference, we performed a numerical analysis prior to the experimental work. For simplicity, our numerical analysis is limited to a $1 \mathrm{D}$ lin $\perp$ lin optical lattice [1] and a $J_{g}=1 / 2 \rightarrow J_{e}=3 / 2$ atom. This lattice beam geometry is the $1 \mathrm{D}$ version of our current experimental setup, and corresponds to two counterpropagating laser beams with orthogonal linear polarizations. In this case there are only two ground state sublevels $|g, \pm\rangle$, which correspond to two sinusoidal optical potentials $U_{ \pm}(z)$ in phase opposition. These potentials are shown in Fig. 2] together with a (stochastic) optical pumping process which transfers an atom from one potential to the other inducing fluctuations in the atomic dynamics. The Fokker-Plancktype equation describing the time evolution of the semiclassical phase-space distribution has been derived in Ref. 14]. As also shown there, this equation can be efficiently integrated by using Monte Carlo simulations techniques.

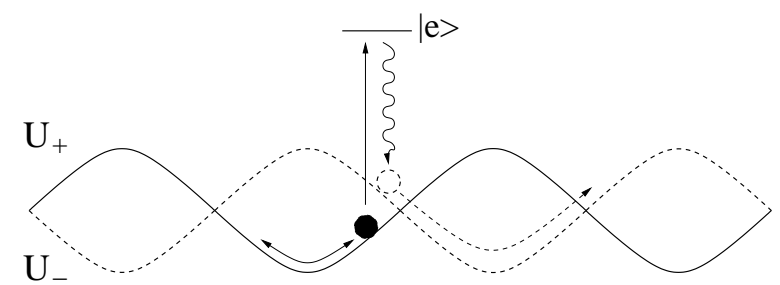

FIG. 2: Optical potentials $\mathrm{U}_{ \pm}$for a $J_{g}=1 / 2 \rightarrow J_{e}=3 / 2$ atom in a $1 \mathrm{D}$ lin $\perp$ lin optical lattice. A (stochastic) process of optical pumping transferring, via an excited state, an atom from a potential to the other one is also shown. The filled (empty) circle represents the atom in the $|g,+\rangle(|g,-\rangle)$ state.

In our calculations a phase modulation of one of the lattice beams of the form of Eq. (2) with $\phi=\pi / 2$ is included to generate the appropriate ac force. We made Monte Carlo simulations for the atomic dynamics and derived the mean atomic velocity as a function of the amplitude of the phase modulation, for a given optical potential depth and modulation frequency, at various optical pumping rates. From the numerical results, shown in Fig. 3] it appears that a current is generated, a result which stimulates our experimental work. The dependence of the current amplitude on the optical pumping rate evidenced in the numerical simulations will be discussed once the experimental findings are presented. It should be noted that in reporting the numerical results (and the same will apply to the experimental findings) we do not distinguish between velocities in the laboratory frame, defined by the $z$ coordinate, and in the accelerated frame, defined by $z^{\prime}=z-\alpha(t) /(2 k)$, in which the optical potential is stationary. This because the two velocities coincide once averaged over time scales $T$ much larger than the ac forces period. The typical frequency $\omega$ considered in this work is about $100 \mathrm{kHz}$, while the typical averaging time is larger than $1 \mathrm{~ms}$, so the average velocities in the laboratory and accelerated frames are equal.

In our experiment cesium atoms are cooled and trapped in a magneto-optical trap. At a given instant the trap is switched off and the four lattice beams are turned on. The lattice fields are red detuned with respect to the $F_{g}=4 \rightarrow F_{e}=5 \mathrm{D}_{2}$ line. The phase modulation $\alpha(t)$, see Eq. (2), of beam 1 is then slowly turned on. The modulating signal is obtained by adding the output signals of two phase-locked oscillators, with oscillation frequencies $\omega$ and $2 \omega$ and phase difference $\phi=\pi / 2$. We observed the motion of the atoms in the lattice by direct imaging of the atomic cloud with a CCD camera. Consistenly with previous work [4, 13, 15] we observed directed motion of the atoms through the lattice along the $z$ direction, following the time-symmetry breaking. We observed a uniform motion of the center of mass of the atomic cloud, and we derived from the experimental data a mean atomic velocity. Several sets of measurements were made for different choices of the lattice beams' parameters (intensity $I$ 
and detuning $\Delta$ from atomic resonance), and modulation amplitude $\alpha_{0}$. The lattice beams' intensity and detuning were varied simultaneously to keep constant the depth of the optical potential $U_{0} \propto I / \Delta$, as verified by pumpprobe spectroscopy, while changing the optical pumping rate $\Gamma^{\prime} \propto I / \Delta^{2}$. In this way we have measured, for a given optical potential, the average atomic velocity as a function of the modulation amplitude, i.e. as a function of the ac force amplitude, for different optical pumping rates, i.e. for different noise levels.

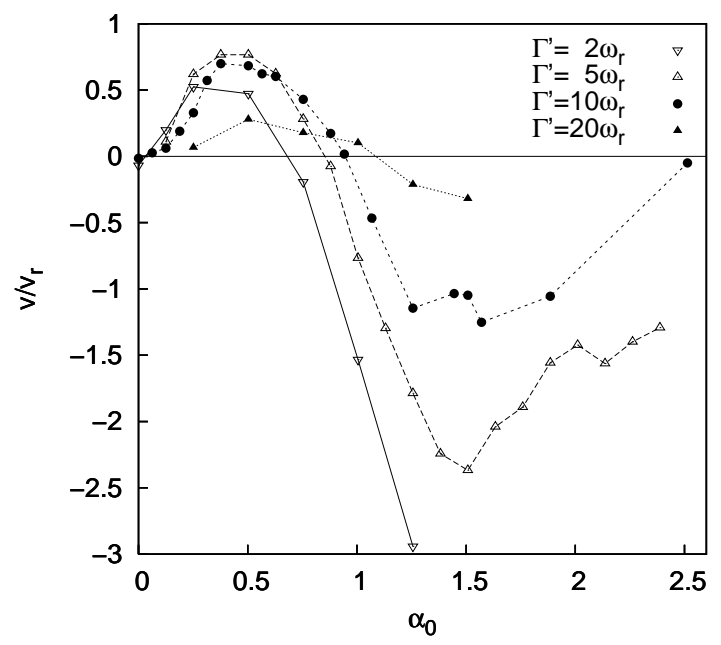

FIG. 3: Results of semiclassical Monte Carlo simulations for a sample of $n=10^{4}$ atoms in a $1 \mathrm{D}$ lin $\perp$ lin optical lattice. The average atomic velocity, in units of the recoil velocity $v_{r}=\hbar k / m$, is shown as a function of the amplitude of the phase modulation. Different data sets correspond to different optical pumping rates $\Gamma$ '. The lines are guides for the eyes. The parameters of the calculations are: the depth of the optical potential is $U_{0}=200 / 3 \cdot E_{r}$, with $E_{r}$ the recoil energy; the coefficients of the harmonics composing the phase modulation, see Eq. (2), are equal: $A=B=1$, the relative phase between the two harmonics is $\phi=\pi / 2$, and the frequency of the modulation is $\omega=0.92 \cdot \omega_{v}$, where $\omega_{v}$ is the vibrational frequency of the atoms at the bottom of the well.

Results of our measurements are reported in Fig. 4 The experimental data show the same behavior as our numerical results (see Fig. 3). For small amplitudes of the ac force the average atomic velocity is an increasing function of the force amplitude, with the atoms moving in the positive $z$ direction. At larger amplitudes of the ac force the velocity decreases, and a current reversal is observed, with the atomic cloud moving in the negative $z$ direction. We note that the numerical simulations (see Fig. (3) show that at large ac forces amplitude the current reaches a maximum and then decreases at increasing amplitude of the ac forces. This has a simple explanation [5]: for very large amplitudes of the applied ac forces the influence of the periodic potential on the atomic dynamics becomes negligible, and the current of atoms decreases.
This behavior has not been observed in the experiment because, due to technical limitations, we were not able to explore modulation amplitudes $\alpha_{0}$ large enough.
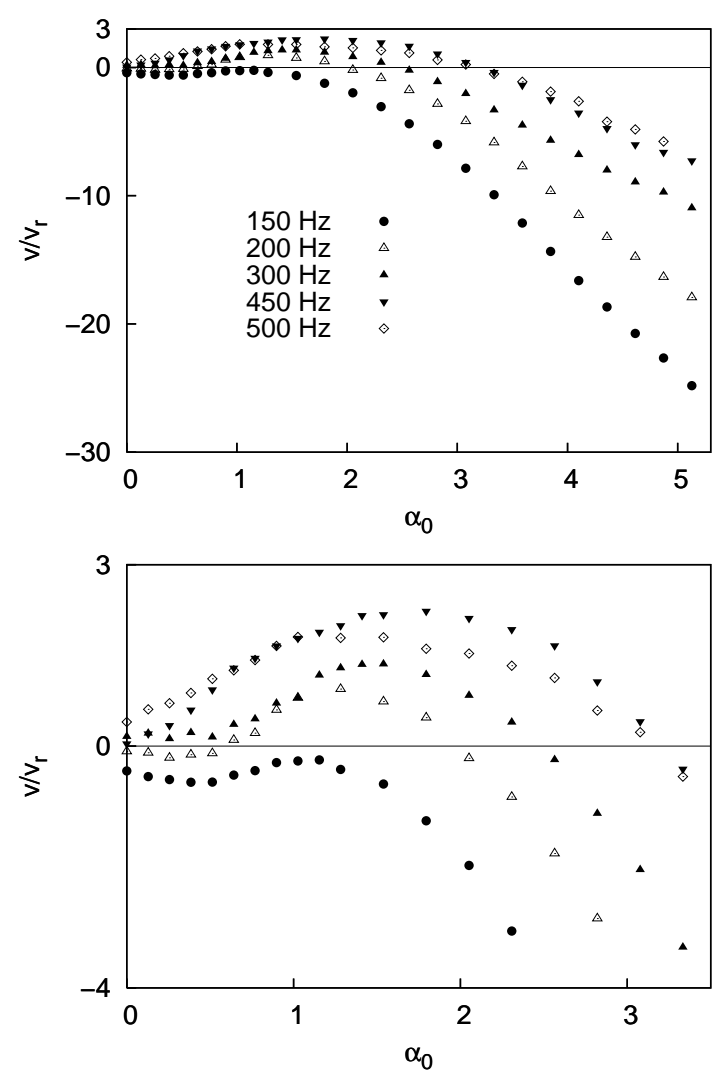

FIG. 4: Experimental results for the mean atomic velocity as a function of the amplitude of the phase modulation. The top graph includes all our experimental results, while the bottom one evidences the region of small ac forces. The optical potential is the same for all measurements, and corresponds to a vibrational frequency $\omega_{v}=2 \pi \cdot 144 \mathrm{kHz}$. Different data sets correspond to different detunings $\Delta$, i.e. to different optical pumping rates as the optical potential is kept constant. The data are labelled by the quantity $\Gamma_{s}=\left[\omega_{v} /(2 \pi)\right]^{2} / \Delta$ which is proportional to the optical pumping rate. The modulation frequency is $\omega=2 \pi \cdot 130 \mathrm{kHz}$. The values for the velocity are expressed in terms of the recoil velocity $v_{r}$, equal to 3.52 $\mathrm{mm} / \mathrm{s}$ for the the $\mathrm{Cs} \mathrm{D}_{2}$ line. Each datapoint corresponds to an average over five images of the atomic cloud. In plotting the data sets for the different pumping rates, adjacent averaging on each data set has also been made. As unwanted consequence, a small vertical shift (positive or negative depending on the data set) for the datapoints close to $\alpha_{0}=0$ has been introduced by the averaging procedure. Errorbars are of the order of the size of the datapoints.

It is important to distinguish the different mechanisms leading to current generation. For large amplitudes of the applied force, the motion can be attributed to deterministic forces and corresponds to force rectification by harmonic mixing: in a nonlinear medium the two har- 
monics, of frequency $\omega$ and $2 \omega$ and phase difference $\phi$, are mixed and result in a rectified force $\bar{F} \propto \sin \phi$. In our experiment the nonlinearity of the medium is the nonlinearity of the optical potential. In this regime the noise does not play any constructive role in the generation of the current of atoms. On the contrary, the noise disturbs the process of rectification of the forces, and, as it appears from our experimental data, for large ac forces the average atomic velocity decreases for increasing optical pumping rate, i.e. for increasing level of the noise, in agreement with our numerical simulations (Fig. 3). We therefore conclude that for large applied forces our experimental realization does not correspond to a Brownian motor.

Consider now the case of small applied ac forces. As evidenced by our experimental data (Fig. [4 bottom) the dependence of the average atomic velocity on the optical pumping rate is completely different from the one observed at large ac forces. For small values of the optical pumping rate the current amplitude is an increasing function of the pumping rate, and the current vanishes in the limit of vanishing optical pumping rate, as shown by the filled circles datapoints of Fig. 团 (bottom) which correspond to the smallest value of the optical pumping rate explored in our experiment. Finally, at larger pumping rates the current reaches a maximum and then decreases. This stochastic resonance-like behavior is the demonstration that in the reverse current regime our optical lattice acts as a fluctuations rectifier, i.e. we realized a Brownian motor.

In conclusion, in this work we presented the realization of a Brownian motor by using cold atoms in a dissipative optical lattice as a model system. We considered a spatially symmetric optical lattice, and we broke the time-symmetry of the system by applying an appropri- ate zero mean ac force. A current of atoms is generated as a result of the time-symmetry breaking. We identify two different regimes, depending on the amplitude of the applied ac force. For large amplitudes the current is produced by deterministic forces, and can be traced back to the rectification by harmonic mixing of the applied oscillating forces. In this regime the noise acts as a disturbance for the rectification process, and correspondingly the current amplitude is a decreasing function of the noise level. At small amplitude of the ac force, the current is reversed and is due to the rectification of fluctuations, with the current amplitude showing a stochastic resonance-like dependence on the noise level. This corresponds to the realization of a Brownian motor.

The present work also shows the important role that optical lattices can play in generating models for statistical physics. With respect to solid state devices [8] or laser tweezer setups [9], optical lattices offer a wider tunability. The depth of the defect-free optical potentials can be controlled by simply changing the laser parameters, and by changing the arrangement and the number of laser beams both periodic and quasi-periodic lattices of different dimension, lattice spacing and lattice geometry can be obtained. Furthermore, the laser parameters also allow a precise control of the optical pumping rate, which can be varied over a very broad range, and also eventually be completed suppressed. This allows the investigation of the vast field of noise-induced phenomena. Among the phenomena identified theoretically that can be explored by our current set-up we mention: dissipation-induced symmetry breaking [7], giant acceleration of free diffusion in tilted lattices [16], Levy walks and anomalous diffusion [17]. Furthermore, in the limit of far detuning from atomic resonance, i.e. by suppressing dissipation, deterministic (chaotic) ratchets [18] can be investigated.
[1] K. Wiesenfeld and F. Moss, Nature 373, 33 (1995); L. Gammaitoni et al., Rev. Mod. Phys. 70, 223 (1998); M.I. Dykman and P.V.E. McClintock, Nature 391, 344 (1998).

[2] R.D. Astumian and P. Hänggi, Phys. Today 55, 33 (2002); P. Reimann, Phys. Rep. 361, 57 (2002); P. Reimann and P. Hänggi, Appl. Phys. A 75, 169 (2002); see also P.V.E. McClintock, Nature 401, 23 (1999).

[3] M.O. Magnasco, Phys. Rev. Lett. 71, 1477 (1993).

[4] A. Adjari et al., J. Phys. I (France) 4, 1551 (1994); M.C. Mahato and A.M. Jayannavar, Phys. Lett. A $\mathbf{2 0 9}$, 21 (1995); D.R. Chialvo and M.M. Millonnas, ibid. 209, 26 (1995); M.I. Dykman et al., Phys. Rev. Lett. 79, 1178 (1997); S. Flach, O. Yevtushenko and Y. Zolotaryuk, ibid. 84, 2358 (2000); S. Denisov et al., Phys. Rev. E 66, 041104 (2002); M.V. Fistul, A.E. Miroshnichenko and S. Flach, Phys. Rev. B 68, 153107 (2003); I. Goychuk and P. Hänggi, Europhys. Lett. 43, 503 (1998).

[5] R. Bartussek, P. Hänggi and J.G. Kissner, Europhys. Lett. 28, 459 (1994).

[6] Ya.M. Blanter and M. Büttiker, Phys. Rev. Lett. 81,
4040 (1998).

[7] P. Reimann, Phys. Rev. Lett. 86, 4992 (2001); O. Yevtushenko et al., Europhys. Lett. 54, 141 (2001).

[8] H. Linke et al., Europhys. Lett. 44, 341 (1998); S. Weiss et al., ibid. 51, 499 (2000).

[9] T. Harada and K. Yoshikawa, Phys. Rev. E 69, 031113 (2004).

[10] F. Jülicher, A. Ajdari and J. Prost, Rev. Mod. Phys. 69, 1269 (1997).

[11] For a recent review of optical lattices, see G. Grynberg and C. Mennerat-Robilliard, Phys. Rep. 355, 335 (2001).

[12] K.I. Petsas, A.B. Coates, and G. Grynberg, Phys. Rev. A 50, 5173 (1994).

[13] M. Schiavoni et al., Phys. Rev. Lett. 90, 094101 (2003).

[14] K.I. Petsas, G. Grynberg and J.-Y. Courtois, Eur. Phys. J. D 6, 29 (1999).

[15] M.I. Dykman et al., Chaos 11, 587 (2001).

[16] P. Reimann et al., Phys. Rev. Lett. 87, 010602 (2001)

[17] S. Marksteiner, K. Ellinger, and P. Zoller, Phys. Rev. A 53, 3409 (1996).

[18] P. Jung, J.G. Kissner and P. Hänggi, Phys. Rev. Lett. 
76, 3436 (1996). 